\title{
Local estimates for minimizers of some convex integral functional of the gradient and the Strong Maximum Principle*
}

\author{
Vladimir V. Goncharov ${ }^{\dagger}$ and Telma J. Santos \\ CIMA-UE, Rua Romão Ramalho 59, 7000-671, Évora, Portugal ${ }^{\ddagger}$
}

\begin{abstract}
We consider a class of convex integral functionals with lagrangeans depending only on the gradient and satisfying a generalized symmetry assumption, which includes as a particular case the rotational symmetry. Adapting the method by A. Cellina we obtain a kind of local estimates for minimizers in the respective variational problems, which is applied then to deduce some versions of the Strong Maximum Principle in the variational setting.

Key words: Strong Maximum Principle, comparison theorems, convex variational problems, Minkowski functional

Mathematical Subject Classification (2000): 49J10, 49J53, 49N15
\end{abstract}

\section{Introduction}

It is well known that the minimizers of an integral functional on a set of Sobolev functions satisfy often the same properties as the solutions of (ordinary or partial) differential equations (such as regularity properties, comparison theorems and so on), even if the classic necessary optimality conditions are not valid. One of these properties is the so called Strong Maximum Principle formulated in the classic form for harmonic functions (each nonconstant harmonic function can not admit its maximal or minimal value in interior of the domain), and generalized then for solutions of a large class of elliptic (as well as parabolic) equations (see, e.g., $[6,9,10]$ and the bibliography therein).

Taking into account that an elliptic equation can be seen as the EulerLagrange equation in the variational problem for some energy functional (such

\footnotetext{
${ }^{*}$ Work supported by: projecto POCI/MAT/56727/2004 and Financiamento Programático Especial do CIMA-UE (Centro de Investigação em Matemática e Aplicações da Universidade de Évora, Portugal) both of FCT (Fundação para Ciência e Tecnologia, Portugal)

${ }^{\dagger}$ Corresponding author. Tel.: +351 936514934; Fax: +351 266745393

‡E-mail: goncha@uevora.pt (V. Goncharov); tjfs@uevora.pt (T. Santos)
} 
as, e.g., the Laplace equation $\Delta u=0$ is the necessary and sufficient condition of minimum of the integral $\left.\int_{\Omega}\|\nabla u(x)\|^{2} d x\right)$, in 2000 Arrigo Cellina has formulated (see [1]) the Strong Maximum Principle (the Strong Minimum Principle, in fact) in the variational setting. Namely, a lower semicontinuous convex lagrangean $L: \mathbb{R}^{n} \rightarrow \mathbb{R}^{+} \cup\{+\infty\}$ with $L(0)=0$ is said to satisfy the Strong Maximum Principle (SMP) if for any open bounded connected domain $\Omega \subset \mathbb{R}^{n}$ a nonnegative continuous admissible solution $\bar{u}(\cdot)$ of the problem

$$
\operatorname{Min}\left\{\int_{\Omega} L(\nabla u(x)) d x: u(\cdot) \in u_{0}(\cdot)+W_{0}^{1,1}(\Omega)\right\}
$$

can be equal to zero at some point $x^{*} \in \Omega$ only in the case $\bar{u}(x) \equiv 0$. Recall that admissible solutions of (1.1) are those, which give finite values to the integral. In the case of rotationally invariant lagrangeans, i.e., $L(\xi)=f(\|\xi\|)$ with a lower semicontinuous convex function $f: \mathbb{R}^{+} \rightarrow \mathbb{R}^{+} \cup\{+\infty\}, f(0)=0$, simple geometric necessary and sufficient conditions guaranteeing the validity of SMP were proposed in [1]. Roughly speaking, these conditions require smoothness and strict convexity of the function $f(\cdot)$ at the origin.

Here we assume the lagrangean to be symmetric in a more general sense, namely, $L(\xi)=f\left(\rho_{F}(\xi)\right)$, where $F \subset \mathbb{R}^{n}$ is a compact convex set containing the origin in its interior, and $\rho_{F}(\cdot)$ means the Minkowski functional (gauge function) associated to $F$,

$$
\rho_{F}(\xi):=\inf \{\lambda>0: \xi \in \lambda F\} .
$$

Variational problems with such type integrands were recently considered in [3], where the authors proved a comparison theorem in the case of the strictly convex gauge $F$. They constructed a comparison function as a solution of the associated Euler-Lagrange equation written in the classic divergence form, essentially using for this the differentiability of the dual Minkowski functional $\xi \mapsto \rho_{F^{0}}(\xi)$, or, in other words, the strict convexity of $F$.

We do not suppose instead the set $F$ to be strictly convex. Based on the duality arguments of Convex Analysis we obtain first (Section 3) some kind of estimates of minimizers in (1.1) near the points, which are distant from their local extremums, emphasizing specially the one-dimensional case. Then, in Section 4, we show that the Cellina's conditions [1] are sufficient and necessary for the validity of the Strong Maximum (Minimum) Principle with no any supplementary assumptions on $F$.

On the other hand, SMP can be extended as follows. Let us fix a continuous function $\hat{u}(\cdot)$ (called further test function) giving minimum to the functional $\int_{\Omega} L(\nabla u(x)) d x$ on $\hat{u}(\cdot)+W_{0}^{1,1}(\Omega)$ for each appropriate domain $\Omega \subset \mathbb{R}^{n}$. We say that the lagrangean $L(\cdot)$ satisfies the generalized Strong Minimum (Maximum) Principle w.r.t. $\hat{u}(\cdot)$ if for any $\Omega \subset \mathbb{R}^{n}$ (belonging to a suitable class of regions) each other minimizer $u(\cdot)$ with $u(x) \geq \hat{u}(x)$ (respectively, with $u(x) \leq \hat{u}(x)$ ), $x \in \Omega$, admitting the same local minimal (respectively, maximal) values as 
$\hat{u}(\cdot)$ at common points, necessarily coincides with $\hat{u}(\cdot)$. (Observe that in the traditional version of SMP mentioned above the test function is the identically zero function, obviously a solution of the variational problem (1.1)). It turns out that such generalized property holds for the lagrangean $L(\xi)=f\left(\rho_{F}(\xi)\right)$, where the gauge is supposed to be smooth and one of the Cellina's hypotheses on $f(\cdot)$ may fail. Namely, we prove in Section 5 a "one-point" version of the generalized SMP with $\hat{u}(\cdot)$ associated to the dual gauge function $\rho_{F^{0}}(\xi)$, assuming the domain $\Omega$ to be bounded and star-shaped w.r.t. the (unique) extremum point of $\hat{u}(\cdot)$ (in particular, $\Omega$ can be convex). Moreover, we give an example showing that a kind of star-shapeness of $\Omega$ is essential for the validity of this property. Finally, in Section 6 we extend SMP to the case of a test function $\hat{u}(\cdot)$ having a finite number of local minimum (maximum) points.

\section{Preliminaries}

Here we introduce the notations and definitions being used in the paper, and formulate the main standing assumptions.

Let $f: \mathbb{R}^{+} \rightarrow \mathbb{R}^{+} \cup\{+\infty\}$ be a lower semicontinuous convex function not equal to zero identically but with $f(0)=0$, and $F \subset \mathbb{R}^{n}, n \in \mathbb{N}$, be a convex closed bounded set with $0 \in \operatorname{int} F$ (interior of $F$ ). Given an open bounded region $\Omega \subset \mathbb{R}^{n}$ we are interested in the behaviour of continuous functions, which minimize the integral

$$
\int_{\Omega} f\left(\rho_{F}(\nabla u(x))\right) d x
$$

among all $u(\cdot) \in W^{1,1}(\Omega)$ with the same boundary data. The function $f(\cdot)$ is clearly nondecreasing that implies convexity of the lagrangean $f \circ \rho_{F}$ in (2.1). In what follows we assume also that $\operatorname{dom} f:=\{t: f(t)<+\infty\} \neq\{0\}$. This does not diminish generality since otherwise each admissible minimizer of the functional (2.1) is constant, and all the results below hold trivially.

Together with the Minkowski functional $\rho_{F}(\xi)$ defined by (1.2) we introduce the support function $\sigma_{F}: \mathbb{R}^{n} \rightarrow \mathbb{R}^{+}, \sigma_{F}(v):=\sup \{\langle v, \xi\rangle: \xi \in F\}$, and recall that

$$
\rho_{F}(\xi)=\sigma_{F^{0}}(\xi), \quad \xi \in \mathbb{R}^{n},
$$

where $F^{0}:=\left\{v \in \mathbb{R}^{n}: \sigma_{F}(v) \leq 1\right\}$ is the polar set. Here $\langle\cdot, \cdot\rangle$ means the inner product in $\mathbb{R}^{n}$ (the norm is denoted by $\|\cdot\|$ ). By the standing hypotheses on $F$ we have obviously that $\left(F^{0}\right)^{0}=F$, and it follows from $(2.2)$ that

$$
\frac{1}{\|F\|}\|\xi\| \leq \rho_{F}(\xi) \leq\left\|F^{0}\right\|\|\xi\|, \quad \xi \in \mathbb{R}^{n},
$$

where $\|F\|:=\sup \{\|\xi\|: \xi \in F\}$.

Furthermore, for a convex lower semicontinuous function $L: \mathbb{R}^{n} \rightarrow \mathbb{R}^{+} \cup$ $\{+\infty\}$ (in particular, for $L=f \circ \rho_{F}$ ) we denote by $L^{*}$ the Legendre-Fenchel 
conjugate and by $\partial L(\xi)$ the subdifferential of $L$ at $\xi$ in the sense of Convex Analysis. These operations can be applied to the function $f(\cdot)$ itself as far as it is somehow extended to the whole real line, e.g., by setting $f(t):=+\infty$ for $t<0$. It is well known that the relations $v \in \partial L(\xi)$ and $\xi \in \partial L^{*}(v)$ are equivalent, and for each $\xi \in \mathbb{R}^{n}$ the equality

$$
\partial \rho_{F}(\xi)=\mathbf{N}_{F}\left(\frac{\xi}{\rho_{F}(\xi)}\right) \cap \partial F^{0}
$$

holds (see, e.g., [5, Corollary 2.3]). Here $\partial F^{0}$ stands for the boundary of the set $F^{0}$, and $\mathbf{N}_{F}(\xi)$ is the normal cone to the set $F$ at $\xi \in \partial F$, i.e., the subdifferential of the indicator function $\mathbf{I}_{F}\left(\mathbf{I}_{F}(x)\right.$ is equal to 0 on $F$ and to $+\infty$ elsewhere).

For the basic facts of Convex Analysis we refer to [11] or to [8]. Let us recall only a pair of dual properties, which will be used in the sequel (see Sections 5 and 6). We say that the set $F$ is smooth (has the smooth boundary) if for each $\xi \in \partial F$ there exists a unique $v \in \mathbf{N}_{F}(\xi)$ with $\|v\|=1$. By (2.4) this property is equivalent to the differentiability of $\rho_{F}(\cdot)$ at each $\xi \neq 0$. On the other hand, $F$ is said to be rotund (strictly convex) if for each $x, y \in \partial F, x \neq y$, and $0<\lambda<1$ we have $(1-\lambda) x+\lambda y \in \operatorname{int} F$. Given $r>0$ and $0<\alpha<\beta<1$ let us define the following modulus of rotundity:

$$
\begin{aligned}
\mathfrak{M}_{F}(r ; \alpha, \beta):=\inf \left\{1-\rho_{F}(\xi+\lambda(\eta-\xi)):\right. \\
\left.\quad \xi, \eta \in \partial F, \rho_{F}(\xi-\eta) \geq r ; \alpha \leq \lambda \leq \beta\right\} .
\end{aligned}
$$

By the compactness argument in a finite-dimensional space the set $F$ is rotund if and only if $\mathfrak{M}_{F}(r ; \alpha, \beta)>0$ for all $r>0$ and $0<\alpha<\beta<1$. The rotundity can be also interpreted in terms of nonlinearity of the gauge function $\rho_{F}(\cdot)$. Namely, $F$ is rotund iff the equality $\rho_{F}(x+y)=\rho_{F}(x)+\rho_{F}(y)$ holds only in the case when $x=\lambda y$ with $\lambda \geq 0$. It is well-known that the polar set $F^{0}$ is rotund if and only if $F$ is smooth.

Since $f \neq 0$ and $\operatorname{dom} f \neq\{0\}$, by the duality argument we also have $f^{*} \neq 0$, $\operatorname{dom} f^{*} \neq\{0\}$ and $f^{*}(0)=0$. Therefore, due to the elementary properties of convex functions there exist $k, a \in[0,+\infty[$ and $0<b \leq+\infty$ such that

$$
\begin{aligned}
\partial f(0) & =[0, k] ; \\
\partial f^{*}(0) & =\{t: f(t)=0\}=[0, a] ; \\
\operatorname{dom} f^{*} & =[0, b] \quad \text { or }[0, b[) .
\end{aligned}
$$

Let us define the function $\varphi:\left[0, b\left[\rightarrow \mathbb{R}^{+}\right.\right.$by

$$
\varphi(t):=\sup \partial f^{*}(t)<+\infty .
$$

It is nondecreasing by monotonicity of the subdifferential. For the sake of convenience we introduce the number $\gamma_{n, f}$ that equals $k$ whenever $n=1$ and $f$ is not affine in a neighbourhood of zero (i.e., $f(t) / t \neq$ const near 0 ), and $\gamma_{n, f}=0$ in all other cases. 
For an open bounded connected domain $\Omega \subset \mathbb{R}^{n}$ we introduce the functions

$$
\mathfrak{r}^{ \pm}(x)=\mathfrak{r}_{\Omega}^{ \pm}(x):=\sup \left\{r>0: x \pm r F^{0} \subset \Omega\right\}, x \in \Omega .
$$

It is not difficult to see that

$$
\mathfrak{r}^{+}(x) \leq \mathfrak{r}^{+}(y)+\rho_{F^{0}}(y-x)
$$

and

$$
\mathfrak{r}^{-}(x) \leq \mathfrak{r}^{-}(y)+\rho_{F^{0}}(x-y)
$$

for all $x, y \in \Omega$, which together with (2.3) imply, in particular, the Lipschitz continuity of $\mathfrak{r}^{ \pm}: \Omega \rightarrow \mathbb{R}^{+}$. Given $x_{0} \in \Omega$ the set

$$
\operatorname{St}\left(x_{0}\right)=\operatorname{St}_{\Omega}\left(x_{0}\right):=\left\{x:\left[x_{0}, x\right] \subset \Omega\right\}
$$

is said to be the star in $\Omega$ associated to the point $x_{0}$. Here

$$
\left[x_{0}, x\right]:=\left\{(1-\lambda) x_{0}+\lambda x: 0 \leq \lambda \leq 1\right\}
$$

is the closed segment connecting $x_{0}$ and $x$. It follows immediately from (2.9) that $\operatorname{St}_{\Omega}\left(x_{0}\right)$ is open. As usual the region $\Omega$ is said to be star-shaped w.r.t. $x_{0} \in \Omega$ if $\Omega=\mathrm{St}\left(x_{0}\right)$. For instance, a convex domain $\Omega$ is star-shaped w.r.t. each point $x \in \Omega$. We say also that $\Omega$ is densely star-shaped w.r.t. $x_{0}$ if $\Omega \subset \overline{\operatorname{St}\left(x_{0}\right)}$, where overbar means the closure in $\mathbb{R}^{n}$.

\section{Local estimates}

Under the notations and the standing assumptions given in Section 2 we can formulate now the basic result on a priori estimates of minimizers close to their non extremum points. Roughly speaking, we affirm that if $\bar{x} \in \Omega$ is not a point of local minimum (maximum) of a solution $\bar{u}(\cdot)$ to the variational problem (1.1) with $L=f \circ \rho_{F}$, then $\bar{u}(\cdot)$ is bounded from below (above) near $\bar{x}$ by a linear function associated to $\rho_{F^{0}}$. This function thus controls the deviation of the value $\bar{u}(x)$ from the respective extremal level.

Theorem 1 Let $\Omega \subset \mathbb{R}^{n}$ be an open bounded region. Then for each continuous admissible minimizer $\bar{u}(\cdot)$ of the functional (2.1) on $u_{0}(\cdot)+W_{0}^{1,1}(\Omega)$ the following statements hold, where the number $a>0$ and the nondecreasing function $\varphi(\cdot)$ are taken from (2.6) and from (2.7), respectively.

(i) Assume that a point $\bar{x} \in \Omega$ and numbers $\beta>0$ and $\mu \in \mathbb{R}$ are such that

$$
\bar{u}(x) \geq \mu \quad \forall x \in \bar{x}-\beta F^{0} \subset \Omega
$$

and

$$
\bar{u}(\bar{x})>\mu+a \beta .
$$


Then there exists $\eta>0$ such that

$$
\bar{u}(x) \geq \mu+\varphi\left(\gamma_{n, f}+\eta\right)\left(\beta-\rho_{F^{0}}(\bar{x}-x)\right)
$$

for all $x \in \bar{x}-\beta F^{0}$.

(ii) Similarly, if in the place of (3.1) and (3.2) a point $\bar{x} \in \Omega$ and numbers $\beta>0, \mu \in \mathbb{R}$ satisfy the inequalities

$$
\bar{u}(x) \leq \mu \quad \forall x \in \bar{x}+\beta F^{0} \subset \Omega
$$

and

$$
\bar{u}(\bar{x})<\mu-a \beta,
$$

then there exists $\eta>0$ such that

$$
\bar{u}(x) \leq \mu-\varphi\left(\gamma_{n, f}+\eta\right)\left(\beta-\rho_{F^{0}}(x-\bar{x})\right)
$$

for all $x \in \bar{x}+\beta F^{0}$.

Proof. (i) If $k>0$ but $\gamma_{n, f}=0$ (either $n>1$ or $f(\cdot)$ is affine near 0 ) then the statement is trivial because $\varphi(\eta)=0$ for each $0<\eta<k$.

Let us suppose now $k=0$. Then $\gamma_{n, f}=0$ and $\varphi(t)>0$ for all $t>0$. Taking into account the continuity of the function $\bar{u}(\cdot)$ and the upper semicontinuity of $\varphi(\cdot)$ it follows from (3.2) that for some small $\delta>0$ and $\alpha \in] 0, \beta[$ the inequality

$$
\bar{u}(x) \geq \mu+\varphi(t)\left(\beta-\rho_{F^{0}}(\bar{x}-x)\right)
$$

holds whenever $\rho_{F^{0}}(\bar{x}-x) \leq \alpha$ and $0<t \leq \delta$. Let us consider the real-valued function $s \mapsto \varphi\left(\delta\left(\frac{\alpha}{s}\right)^{n-1}\right)$, which is (Riemann) integrable on the interval $[\alpha, \beta]$. Denoting by

$$
R_{\delta}(r):=\mu+\int_{r}^{\beta} \varphi\left(\delta\left(\frac{\alpha}{s}\right)^{n-1}\right) d s,
$$

we deduce from (3.7) that

$$
\bar{u}(x) \geq R_{\delta}\left(\rho_{F^{0}}(\bar{x}-x)\right)
$$

for all $x \in \Omega$ with $\rho_{F^{0}}(\bar{x}-x)=\alpha$. Observe that $R_{\delta}(\beta)=\mu$, and by (3.1) the inequality (3.9) holds for $x \in \Omega$ with $\rho_{F^{0}}(\bar{x}-x)=\beta$. Our goal now is to establish this inequality in the interior of the region

$$
A_{\alpha, \beta}:=\left\{x \in \mathbb{R}^{n}: \alpha \leq \rho_{F^{0}}(\bar{x}-x) \leq \beta\right\}
$$

using its validity on the boundary $\partial A_{\alpha, \beta}$, i.e., to prove a kind of comparison statement. Earlier estimates of this kind were considered, e.g., in [1, 3].

Denote by $S_{\delta}(x):=R_{\delta}\left(\rho_{F^{0}}(\bar{x}-x)\right)$ and assume that the (open) set

$$
U:=\left\{x \in A_{\alpha, \beta}: \bar{u}(x)<S_{\delta}(x)\right\}
$$


is nonempty. Let us extend the Lipschitz continuous function $S_{\delta}: A_{\alpha, \beta} \rightarrow \mathbb{R}^{+}$ to the whole $\bar{\Omega}$ by setting $S_{\delta}(x)=R_{\delta}(\beta)=\mu$ for $x \in \bar{\Omega}$ with $\rho_{F^{0}}(\bar{x}-x)>\beta$, and $S_{\delta}(x)=R_{\delta}(\alpha)$ whenever $\rho_{F^{0}}(\bar{x}-x)<\alpha$. Consider the function $w(x):=$ $\max \left\{\bar{u}(x), S_{\delta}(x)\right\}$, which is equal to $S_{\delta}(x)$ on $U$ and to $\bar{u}(x)$ elsewhere, and let us show that $w(\cdot)$ minimizes the functional $(2.1)$ on $\bar{u}(\cdot)+W_{0}^{1,1}(\Omega)$. The crucial step here is proving the equality

$$
\int_{U}\langle p(x), \nabla \bar{u}(x)-\nabla w(x)\rangle d x=0
$$

for some measurable function $p(x) \in \partial\left(f \circ \rho_{F}\right)(\nabla w(x)), x \in \Omega$.

We construct such $p(\cdot)$ as follows. Since $\nabla w(x)=\nabla \bar{u}(x)$ for a.e. $x \in \Omega \backslash U$, and $\nabla w(x)=\nabla S_{\delta}(x)$ for a.e. $x \in U$ (see [7, p. 50]), we may choose $p(x)$ as an arbitrary measurable selection of the mapping $x \mapsto \partial\left(f \circ \rho_{F}\right)(\nabla \bar{u}(x)) \neq \varnothing$ on $\Omega \backslash U$. On the set $U$, instead, we define $p(x)$ through the differentiability of $S_{\delta}(x)$. Observe first of all that $R_{\delta}^{\prime}(r)=-\varphi\left(\delta\left(\frac{\alpha}{r}\right)^{n-1}\right)$ for all $\alpha<r<\beta$ except for at most a countable number of points. Furthermore, by the Lipschitz continuity of $x \mapsto \rho_{F^{0}}(\bar{x}-x)$ we have that

$$
\nabla S_{\delta}(x)=-R_{\delta}^{\prime}\left(\rho_{F^{0}}(\bar{x}-x)\right) \nabla \rho_{F^{0}}(\bar{x}-x)
$$

for almost each $x \in U$. Due to $(2.4)$ the gradient $\nabla \rho_{F^{0}}(\bar{x}-x)$ belongs to $\partial F$, and, consequently,

$$
\rho_{F}\left(\nabla S_{\delta}(x)\right)=\left|R_{\delta}^{\prime}\left(\rho_{F^{0}}(\bar{x}-x)\right)\right|=\varphi\left(\delta\left(\frac{\alpha}{\rho_{F^{0}}(\bar{x}-x)}\right)^{n-1}\right) .
$$

Hence, by the definition of $\varphi(\cdot)$ we obtain that

$$
\delta\left(\frac{\alpha}{\rho_{F^{0}}(\bar{x}-x)}\right)^{n-1} \in \partial f\left(\rho_{F}\left(\nabla S_{\delta}(x)\right)\right)
$$

for a.e. $x \in U$. On the other hand (see (2.4) and (3.11)), $\nabla S_{\delta}(x)$ is a normal vector to $F^{0}$ at the point $\frac{\bar{x}-x}{\rho_{F^{0}}(\bar{x}-x)}$ that means (see also $(2.2)$ )

$$
\left\langle\nabla S_{\delta}(x), \frac{\bar{x}-x}{\rho_{F^{0}}(\bar{x}-x)}\right\rangle=\sigma_{F^{0}}\left(\nabla S_{\delta}(x)\right)=\rho_{F}\left(\nabla S_{\delta}(x)\right),
$$

or, in the dual form,

$$
\frac{\bar{x}-x}{\rho_{F^{0}}(\bar{x}-x)} \in \mathbf{N}_{F}\left(\frac{\nabla S_{\delta}(x)}{\rho_{F}\left(\nabla S_{\delta}(x)\right)}\right) \cap \partial F^{0}=\partial \rho_{F}\left(\nabla S_{\delta}(x)\right) .
$$

Recalling (3.12) let us define the (continuous) function

$$
p(x):=\delta\left(\frac{\alpha}{\rho_{F^{0}}(\bar{x}-x)}\right)^{n-1} \frac{\bar{x}-x}{\rho_{F^{0}}(\bar{x}-x)},
$$


which is a selection of $x \mapsto \partial\left(f \circ \rho_{F}\right)\left(\nabla S_{\delta}(x)\right)$ almost everywhere in $U$.

In order to prove (3.10) we apply the polar coordinates $r=\|x-\bar{x}\|$ and $\omega=(x-\bar{x}) /\|x-\bar{x}\|$, observing that for each $\omega,\|\omega\|=1$, on the boundary of the (open) linear set $l_{\omega}:=\{r \in] \alpha, \beta[:(r, \omega) \in U\}$ the equality $\bar{u}(x)=S_{\delta}(x)$ holds. Therefore,

$$
\int_{l_{\omega}}\left\langle\omega, \nabla \bar{u}(x)-\nabla S_{\delta}(x)\right\rangle d r=\int_{l_{\omega}} \frac{d}{d r}\left(\bar{u}(x)-S_{\delta}(x)\right) d r=0,
$$

and by using Fubini theorem, we obtain

$$
\begin{aligned}
& \int_{U}\left\langle p(x), \nabla \bar{u}(x)-\nabla S_{\delta}(x)\right\rangle d x= \\
= & -\delta \alpha^{n-1} \int_{\|\omega\|=1} \frac{d \omega}{\rho_{F^{0}}^{n}(-\omega)} \int_{l_{\omega}} \frac{1}{r^{n}}\left\langle r \omega, \nabla \bar{u}(x)-\nabla S_{\delta}(x)\right\rangle r^{n-1} d r=0,
\end{aligned}
$$

and the equality (3.10) is proved.

In the next step let us consider the inequality

$$
f\left(\rho_{F}(\nabla \bar{u}(x))\right)-f\left(\rho_{F}(\nabla w(x))\right) \geq\langle p(x), \nabla \bar{u}(x)-\nabla w(x)\rangle,
$$

which holds almost everywhere on $\Omega$ by the choice of $p(\cdot)$. Since $\bar{u}(\cdot)$ is a minimizer of $(2.1)$ on $u_{0}(\cdot)+W_{0}^{1,1}(\Omega)$, it follows from (3.10) and (3.14) that $w(\cdot)$ is a minimizer as well, and

$$
\int_{\Omega} f\left(\rho_{F}(\nabla \bar{u}(x))\right) d x=\int_{\Omega} f\left(\rho_{F}(\nabla w(x))\right) d x .
$$

Furthermore, (3.14) together with (3.15) and (3.10) imply that

$$
f\left(\rho_{F}(\nabla \bar{u}(x))\right)-f\left(\rho_{F}(\nabla w(x))\right)=\langle p(x), \nabla \bar{u}(x)-\nabla w(x)\rangle
$$

for a.e. $x \in \Omega$.

Let $E \subset U$ be a set of null Lebesgue measure such that for all $x \in U \backslash E$ the equality (3.16) takes place, and the gradient $\nabla \sigma_{F}(\bar{x}-x)$ (coinciding, by homogeneity, with $\nabla \sigma_{F}(p(x))$, see (3.13)) exists. In accordance with (3.16) both $\nabla \bar{u}(x)$ and $\nabla w(x)$ belong to $\partial\left(f \circ \rho_{F}\right)^{*}(p(x))=\partial\left(f^{*} \circ \sigma_{F}\right)(p(x))$, and for each $x \in U \backslash E$ the latter subdifferential admits the form

$$
\partial f^{*}\left(\rho_{F^{0}}(p(x))\right) \nabla \sigma_{F}(\bar{x}-x),
$$

where (see (3.13))

$$
\rho_{F^{0}}(p(x))=\delta\left(\frac{\alpha}{\rho_{F^{0}}(\bar{x}-x)}\right)^{n-1} .
$$


Notice that there is an at most a countable family of disjoint open intervals $J_{1}, J_{2}, \ldots \subset[\alpha, \beta]$ such that the real function $f(\cdot)$ is affine on each $J_{i}$ with a slope $\tau_{i}>0, i=1,2, \ldots$. In other words, $\tau_{i}$ are discontinuity points of $\varphi(\cdot)$. Denoting by $E_{i}:=\bar{x}-\alpha\left(\frac{\delta}{\tau_{i}}\right)^{\frac{1}{n-1}} \partial F^{0}$ we see that for all $x \notin \bigcup_{i=1}^{\infty} E_{i}$ (the set of null measure) $\partial f^{*}\left(\rho_{F^{0}}(p(x))\right)$ is a singleton. Hence, $\nabla w(x)=\nabla \bar{u}(x)$ for a.e. $x \in \Omega$ contradicting the assumption $U \neq \varnothing$. Thus, we have proved the inequality (3.9) on $\left\{x \in \Omega: \alpha \leq \rho_{F^{0}}(\bar{x}-x) \leq \beta\right\}$. Combining (3.9) and (3.8) by the mean value theorem we obtain (3.3) with $\eta=\delta\left(\frac{\alpha}{\beta}\right)^{n-1}$.

Finally, assume that $\gamma_{n, f}=k>0$. In this case $n=1$, the function $f(\cdot)$ admits the positive slope at zero but it is not affine near 0 (consequently, $\varphi(k)=$ 0 ), and obviously $a=0$. Then, for a given $\beta>0$ with $\bar{x}-\beta F^{0} \subset \Omega$ we have $\bar{u}(\bar{x})>\mu+\varphi(k) \beta$, and, by continuity properties of the functions $\bar{u}(\cdot)$ and $\varphi(\cdot)$, there exist $\delta>0$ and $0<\alpha<\beta$ such that

$$
\bar{u}(x) \geq \mu+\varphi(k+t)\left(\beta-\rho_{F^{0}}(\bar{x}-x)\right)
$$

for all $x \in \Omega$ with $\rho_{F^{0}}(\bar{x}-x) \leq \alpha$ and $0<t \leq \delta$. Due to the monotonicity of the subdifferential $\partial f^{*}(\cdot)$ and to the nonaffinity of $f(\cdot)$ in a neighbourhood of zero we can choose $\delta>0$ such that $\partial f^{*}(k+\delta)$ is a singleton (equivalently, $k+\delta$ is a slope of $f(\cdot)$ different from slopes of its affine pieces near zero). Now we can proceed as in the first part of the proof by using the comparison argument with the linear function

$$
R_{\delta}(r):=\mu+\varphi(k+\delta)(\beta-r), \quad \alpha \leq r \leq \beta .
$$

The selection $p(x) \in \partial\left(f \circ \rho_{F}\right)\left(\nabla\left(R_{\delta} \circ \rho_{F^{0}}\right)(\bar{x}-x)\right)$ appearing in the equality (3.10) takes the form

$$
p(x)=(k+\delta) \frac{\bar{x}-x}{\rho_{F^{0}}(\bar{x}-x)} .
$$

Observe that the final part of the proof should be omitted here because the subdifferential $\partial f^{*}\left(\rho_{F^{0}}(p(x))\right)=\partial f^{*}(k+\delta)$ is already a singleton by the construction. Thus we have

$$
\bar{u}(x) \geq \mu+\varphi(k+\delta)\left(\beta-\rho_{F^{0}}(\bar{x}-x)\right)
$$

for all $x \in \Omega$ with $\rho_{F^{0}}(\bar{x}-x) \leq \beta$, and the first part of theorem is proved.

(ii) This can be proved by the same reasoning as (i) with some evident modifications. For instance, the inequality (3.9) here admits the form

$$
\bar{u}(x) \leq \tilde{R}_{\delta}\left(\rho_{F^{0}}(x-\bar{x})\right),
$$

where

$$
\tilde{R}_{\delta}(r):=\mu-\int_{r}^{\beta} \varphi\left(\delta\left(\frac{\alpha}{s}\right)^{n-1}\right) d s .
$$


In the case $a=0$, i.e., when the function $f(\cdot)$ is strictly convex at the origin, we immediately obtain a consequence of Theorem 1 , which will be exploited in Section 4 . Here $\mathfrak{r}^{ \pm}(\cdot)$ are the functions defined by $(2.8)$.

Corollary 1 Assume that $a=0$, and one of the following hypotheses holds:

(i) $k=0$;

(ii) $n=1$ and $f(\cdot)$ is not affine near 0 .

If $\bar{u}(\cdot)$ is a continuous admissible minimizer of the functional (2.1) on $u_{0}(\cdot)+$ $W_{0}^{1,1}(\Omega)$, which does not attain its minimal (maximal) value at a point $\bar{x} \in \Omega$, then the whole set $\bar{x}-\mathfrak{r}^{-}(\bar{x}) F^{0}$ (respectively, $\left.\bar{x}+\mathfrak{r}^{+}(\bar{x}) F^{0}\right)$ does not contain in its interior points of minimum (respectively, maximum) of $\bar{u}(\cdot)$.

Proof. It is enough to take $\beta:=\mathfrak{r}^{-}(\bar{x})$ (respectively, $\mathfrak{r}^{+}(\bar{x})$ ) and observe that under the hypothesis (i) or (ii) we have $\varphi\left(\gamma_{n, f}+\eta\right)>0$ for all $\eta>0$. The statement follows now from the estimate (3.3) or (3.6), respectively.

On the other hand, if $a>0$ then the estimates of Theorem 1 can be applied to obtain generalizations of SMP in Sections 5 and 6. Those results are essentially based on the following assertion.

Corollary 2 Assume that $a>0$, and let $\bar{u}(\cdot)$ be a continuous admissible minimizer of (2.1) on $u_{0}(\cdot)+W_{0}^{1,1}(\Omega)$ such that for some $x_{0} \in \Omega$ and $\delta>0$

$$
\bar{u}(x) \geq \bar{u}\left(x_{0}\right)+a \rho_{F^{0}}\left(x-x_{0}\right) \quad \forall x \in x_{0}+\delta F^{0} \subset \Omega .
$$

Then for all $x \in x_{0}+\frac{\delta}{\|F\|\left\|F^{0}\right\|+1} F^{0}$ the equality

$$
\bar{u}(x)=\bar{u}\left(x_{0}\right)+a \rho_{F^{0}}\left(x-x_{0}\right)
$$

holds.

Symmetrically, if

$$
\bar{u}(x) \leq \bar{u}\left(x_{0}\right)-a \rho_{F^{0}}\left(x_{0}-x\right) \quad \forall x \in x_{0}-\delta F^{0} \subset \Omega,
$$

then

$$
\bar{u}(x)=\bar{u}\left(x_{0}\right)-a \rho_{F^{0}}\left(x_{0}-x\right)
$$

for all $x \in x_{0}-\frac{\delta}{\|F\|\left\|F^{0}\right\|+1} F^{0}$.

Proof. We prove the first part of Corollary by using the statement (i) of Theorem 1, while the symmetric assertion can be proved similarly (it is enough only to apply (ii) in the place of (i)).

Assume that for some $\bar{x} \in \Omega$ with $\rho_{F^{0}}\left(\bar{x}-x_{0}\right)<\frac{\delta}{\|F\|\left\|F^{0}\right\|+1}$

$$
\bar{u}(\bar{x})>\mu+a \rho_{F^{0}}\left(\bar{x}-x_{0}\right),
$$


where $\mu:=\bar{u}\left(x_{0}\right)$. Let us choose $\varepsilon>0$ so small that

$$
\rho_{F^{0}}\left(\bar{x}-x_{0}\right)\left(\|F\|\left\|F^{0}\right\|+1\right)+\varepsilon\|F\|\left\|F^{0}\right\|<\delta
$$

and

$$
\bar{u}(\bar{x})>\mu+a\left(\rho_{F^{0}}\left(\bar{x}-x_{0}\right)+\varepsilon\right) .
$$

Set $\beta:=\rho_{F^{0}}\left(\bar{x}-x_{0}\right)+\varepsilon$ and show that $\bar{x}-\beta F^{0} \subset x_{0}+\delta F^{0}$. Indeed, given $y \in \bar{x}-\beta F^{0}$, using the inequalities (2.3) we have

$$
\rho_{F^{0}}(y-\bar{x}) \leq\|F\|\left\|F^{0}\right\| \rho_{F^{0}}(\bar{x}-y) \leq\|F\|\left\|F^{0}\right\|\left(\rho_{F^{0}}\left(\bar{x}-x_{0}\right)+\varepsilon\right),
$$

and it follows from (3.22) that

$$
\rho_{F^{0}}\left(y-x_{0}\right) \leq \rho_{F^{0}}(y-\bar{x})+\rho_{F^{0}}\left(\bar{x}-x_{0}\right)<\delta .
$$

Hence, in particular, $\bar{u}(x) \geq \mu$ for all $x \in \bar{x}-\beta F^{0}$. Combining this inequality with (3.23) by Theorem 1 (i) we find $\eta>0$ such that

$$
\bar{u}(x) \geq \mu+\varphi(\eta)\left(\beta-\rho_{F^{0}}(\bar{x}-x)\right) \quad \forall x \in \bar{x}-\beta F^{0} .
$$

Applying (3.24) to the point $x_{0} \in \bar{x}-\beta F^{0}$, we obtain that

$$
\bar{u}\left(x_{0}\right) \geq \mu+\varphi(\eta) \varepsilon \geq \mu+a \varepsilon>\mu,
$$

which is a contradiction. The equality (3.19) can be extended then to the boundary of $x_{0}+\frac{\delta}{\|F\|\left\|F^{0}\right\|+1} F^{0}$ by continuity of the involved functions.

From the latter part of the proof of Theorem 1(i) it is easy to see that in the case $n=1$, due to the disconnectedness of the annulus $A_{\alpha, \beta}$, the estimates like (3.3) and (3.6) hold without symmetry. To be more precise let us consider a convex (not necessarily even) function $L: \mathbb{R} \rightarrow \mathbb{R}^{+} \cup\{+\infty\}$ with $L(0)=0$ and $0 \in \operatorname{intdom} L$, lower semicontinuous on its domain and such that $L(\cdot)$ is not equal identically to zero on both negative and positive half-lines. In what follows the set of functions with these properties will be denoted by $\mathfrak{L}$. Given $L(\cdot) \in \mathfrak{L}$ it is obvious that the function $L$ decreases on $]-\infty, 0[$ and increases on $] 0,+\infty[$, that there exist $0<b^{ \pm} \leq+\infty$ with $\operatorname{dom} L^{*}=\left\{t:-b^{-}<t<b^{+}\right\}$, where one of the signs " $<$ " (or both of them) can be replaced to " $\leq "$, and that $0 \in \partial L(0)$, $0 \in \partial L^{*}(0)$. Consequently, for some nonnegative (finite) $k^{ \pm}$and $a^{ \pm}$we have $\partial L(0)=\left[-k^{-}, k^{+}\right]$and $\partial L^{*}(0)=\left[-a^{-}, a^{+}\right]$. Similarly to the symmetric case let us introduce the upper semicontinuous nondecreasing function $\varphi:]-b^{-}, b^{+}[\rightarrow$ $\mathbb{R}$ by setting $\varphi(t):=\sup \partial L^{*}(t)$. Observe, moreover, that by monotonicity of the subdifferential one of the numbers $k^{+}$or $a^{+}$(analogously, $k^{-}$or $a^{-}$) is always equal to zero. The statement below contains the one-sided estimates for solutions of the one-dimensional variational problem (1.1). For the sake of simplicity we consider here only the case of local minimum. One easily makes the respective changements when the opposite conditions (of local maximum) take place. 
Theorem 2 Let $L \in \mathfrak{L}$, and let $\bar{u}(\cdot)$ be a continuous admissible minimizer in the problem (1.1). Assume that a point $\bar{x} \in \Omega$ and numbers $\mu \in \mathbb{R}, \beta>0$ are such that

$$
\bar{u}(x) \geq \mu \quad \forall x \in[\bar{x}-\beta, \bar{x}] \subset \Omega
$$

and

$$
\bar{u}(\bar{x})>\mu+a^{+} \beta .
$$

If $L$ is not affine in a right-hand neighbourhood of zero then there exists $\eta>0$ such that the inequality

$$
\bar{u}(x) \geq \mu+\varphi\left(k^{+}+\eta\right)(\beta+x-\bar{x})
$$

holds for all $x$ with $\bar{x}-\beta \leq x \leq \bar{x}$.

Analogously, if $L$ is not affine in a left-hand neighbourhood of zero and the relations (3.25), (3.26) are substituted to the following:

$$
\begin{gathered}
\bar{u}(x) \geq \mu \quad \forall x \in[\bar{x}, \bar{x}+\beta] \subset \Omega ; \\
\bar{u}(\bar{x})>\mu+a^{-} \beta,
\end{gathered}
$$

then for some $\eta>0$ we have

$$
\bar{u}(x) \geq \mu-\varphi\left(-k^{-}-\eta\right)(\beta+\bar{x}-x)
$$

whenever $\bar{x} \leq x \leq \bar{x}+\beta$.

Proof. We use here the same arguments as in the proof of Theorem 1. Let us emphasize only some simplifications occured in the main steps that has a methodical interest.

Let us consider the first case ( $L$ is not affine to the right of the origin and (3.25), (3.26) are fulfiled). We write (3.26) as $\bar{u}(\bar{x})>\mu+\varphi\left(k^{+}\right) \beta$ and by the upper semicontinuity of $\varphi(\cdot)$ choose $\eta>0$ so small that the latter inequality holds true with $\varphi\left(k^{+}+\eta\right)$ in the place of $\varphi\left(k^{+}\right)$. Assume also (see the last part of the proof of Theorem 1(i)) that the subdifferential $\partial L^{*}\left(k^{+}+\eta\right)$ is a singleton, namely, $\partial L^{*}\left(k^{+}+\eta\right)=\left\{\varphi\left(k^{+}+\eta\right)\right\}$. Here we use nonaffinity of $L(\cdot)$ to the right of zero. Defining on $\bar{\Omega}$ the continuous function

$$
R_{\delta}(x):= \begin{cases}\mu & \text { if } \quad x<\bar{x}-\beta, \\ \mu+\varphi\left(k^{+}+\eta\right)(\beta+x-\bar{x}) & \text { if } \quad \bar{x}-\beta \leq x \leq \bar{x}, \\ \mu+\varphi\left(k^{+}+\eta\right) \beta & \text { if } \quad x>\bar{x},\end{cases}
$$

we wish to prove that $\bar{u}(x) \geq R_{\delta}(x)$ for all $x \in[\bar{x}-\beta, \bar{x}]$. If this inequality is violated in some (open) set $U \subset] \bar{x}-\beta, \bar{x}$ [ then by the Newton-Leibnitz formula

$$
\int_{\Omega}\left(\bar{u}^{\prime}(x)-w^{\prime}(x)\right) d x=\int_{U}\left(\bar{u}^{\prime}(x)-R_{\delta}^{\prime}(x)\right) d x=0,
$$

where $w(x):=\max \left\{\bar{u}(x), R_{\delta}(x)\right\}$. Since $k^{+}+\eta \in \partial L\left(R_{\delta}^{\prime}(x)\right)$, we have

$$
L\left(\bar{u}^{\prime}(x)\right)-L\left(w^{\prime}(x)\right) \geq\left(k^{+}+\eta\right)\left(\bar{u}^{\prime}(x)-w^{\prime}(x)\right)
$$


for a.e. $x \in \Omega$ and conclude by (3.29) and by the choice of $\bar{u}(\cdot)$ that $w(\cdot)$ is a minimizer in the problem (1.1) as well. So that the inequality (3.30) becomes equality almost everywhere on $\Omega$. Consequently, both $\bar{u}^{\prime}(x)$ and $w^{\prime}(x)$ belong to $\partial L^{*}\left(k^{+}+\eta\right)$, i.e., $\bar{u}^{\prime}(x)=w^{\prime}(x)=\varphi\left(k^{+}+\eta\right)$ for a.e. $x \in U$ contradicting the assumption that $\bar{u}(x)<R_{\delta}(x)$ on $U$. Thus, the estimate (3.27) takes place.

The right-sided inequality (3.28), where $-\varphi\left(-k^{-}-\eta^{-}\right)>0$, can be obtained similarly by using the behaviour of $L(\cdot)$ to the left of zero.

We are ready now to establish various versions of SMP, starting from the traditional one.

\section{Strong Maximum Principle in the case $a=0$}

Our standing hypothesis here is

$$
\left(\mathbf{H}_{1}\right) \quad \partial L^{*}(0)=\{0\},
$$

which is, obviously, equivalent to $a=0$ when $L=f \circ \rho_{F}$ (i.e., the same hypothesis for the function $f(\cdot))$. In the case $n>1$ also the dual hypothesis

$$
\left(\mathbf{H}_{2}\right) \quad \partial L(0)=\{0\},
$$

which reduces to $k=0$ if $L=f \circ \rho_{F}$, will be used in the sequel. In the asymmetric case $(n=1)$, clearly, $\left(\mathbf{H}_{1}\right) \Leftrightarrow a^{+}=a^{-}=0$ while $\left(\mathbf{H}_{2}\right) \Leftrightarrow k^{+}=$ $k^{-}=0$.

The following statement generalizes the sufficient condition of SMP given in [1] to the case when the set $F$ is not necessarily smooth or strictly convex. As an example of such situation we may point out, e.g., the integral

$$
\int_{\Omega}\left(\sum_{i=1}^{n}\left|\frac{\partial u}{\partial x_{i}}\right|\right)^{2} d x .
$$

Here $f(t)=t^{2}$ and $\rho_{F}(\cdot)$ is the $l_{1}$-norm in $\mathbb{R}^{n}$.

Theorem 3 (Strong Maximum Principle) Assume that one of the following conditions holds:

(i) $n=1$ and $L(\cdot) \in \mathfrak{L}$ is not affine in both left-and right-hand neighbourhoods of zero;

(ii) $n>1$ and the lagrangean $L(\cdot)$ satisfies both hypotheses $\left(\mathbf{H}_{1}\right)$ and $\left(\mathbf{H}_{2}\right)$, being represented as $L=f \circ \rho_{F}$, where $f: \mathbb{R}^{+} \rightarrow \mathbb{R}^{+} \cup\{+\infty\}$ is a convex lower semicontinuous function with $f(0)=0$, and $F \subset \mathbb{R}^{n}$ is a convex closed bounded set with $0 \in \operatorname{int} F$. 
Then for each open bounded connected region $\Omega \subset \mathbb{R}^{n}$ there is no a continuous admissible nonconstant minimizer of

$$
u(\cdot) \mapsto \int_{\Omega} L(\nabla u(x)) d x
$$

on $u_{0}(\cdot)+W_{0}^{1,1}(\Omega)$ (with arbitrary boundary data), which admits its minimal (or maximal) value in $\Omega$.

Proof. Observe first that in the framework of the condition (i) the hypothesis $\left(\mathbf{H}_{1}\right)$ holds automatically, while $\left(\mathbf{H}_{2}\right)$ can be violated. An open bounded connected region in this case is an interval $\Omega=] A, B$ [ with $A<B$. Assuming that $\bar{u}(\cdot)$ is a minimizer of $(4.1)$, and $\bar{x} \in \Omega$ is such that $\bar{u}(\bar{x})>\mu:=\min _{\bar{\Omega}} \bar{u}(x)$, we put $\beta:=\bar{x}-A$, and by Theorem 2 obtain that $\bar{u}(x)>\mu$ for all $x \in] A, \bar{x}$ ] (because in the estimate (3.27) we have $\left.\varphi\left(k^{+}+\eta\right)>0 \forall \eta>0\right)$. Analogously, by using the estimate (3.28) of Theorem 2 we conclude that $\bar{u}(x)>\mu \forall x \in[\bar{x}, B[$. Consequently, there are only two possibilities: either the minimum of $\bar{u}(\cdot)$ is attained in one of the end-points of the segment $[A, B]$ or $\bar{u} \equiv \mu$ on $\Omega$. In the case of maximum the reasoning is similar.

Let us suppose now the condition (ii). Take an arbitrary continuous admissible function $\bar{u}: \Omega \rightarrow \mathbb{R}$ that minimizes the integral (4.1), and let $\mu$ be its minimum on $\bar{\Omega}$. If $\bar{u}(\cdot)$ is not a constant then the (open) set $W:=$ $\{x \in \Omega: \bar{u}(x) \neq \mu\}$ is nonempty. Since $a=0$, it follows from Corollary 1 that $\bar{x}-\beta F^{0} \subset W$ whenever $\bar{x} \in W, 0<\beta<\mathfrak{r}^{-}(\bar{x})(\operatorname{see}(2.8))$.

Fix now $x^{*} \in \bar{W}$ (closure of $W$ in $\Omega$ ). By the continuity let us choose an arbitrary $0<\varepsilon<\frac{1}{2\|F\|} \mathfrak{r}^{-}\left(x^{*}\right)$ so small that $\mathfrak{r}^{-}\left(x^{*}\right) \leq 2 \mathfrak{r}^{-}(x)$ for all $x \in \Omega$ with $\left\|x-x^{*}\right\| \leq \varepsilon$. Let $\bar{x} \in W \cap\left(x^{*}+\varepsilon \bar{B}\right)$. Then $\bar{x} \in W$ and

$$
\rho_{F^{0}}\left(\bar{x}-x^{*}\right) \leq \varepsilon\|F\|<\frac{1}{2} \mathfrak{r}^{-}\left(x^{*}\right) \leq \mathfrak{r}^{-}(\bar{x}) .
$$

Hence, by the above, $x^{*}$ also belongs to $W$. Therefore, $W$ is closed in $\Omega$, implying that $W=\Omega$ because $\Omega$ is connected. Thus, the open region $\Omega$ is free from the points of mimimum of $\bar{u}(\cdot)$. Analogously, $\bar{u}(\cdot)$ being non constant can not attain in $\Omega$ its maximum, and SMP is proved. Notice that here we used the arguments in some sense dual to the classical proof for harmonic functions (see, e.g., $[6$, p. 15]).

The Strong Maximum Principle is not valid in lack of the hypothesis $\left(\mathbf{H}_{1}\right)$ for each $n \geq 1$ because, as shown in [2], there is a lot of (Lipschitz) continuous nonnegative (nonpositive) minimizers of (4.1) with the trivial boundary condition $u_{0}(x)=0$, which touch the zero level at interior points of $\Omega$ as well. If instead $\left(\mathbf{H}_{2}\right)$ is violated then in the case $n>1$ one can construct a counterexample to SMP based on the same arguments as those in [1].

Indeed, fix arbitrary $\bar{x} \in \mathbb{R}^{n}, \mu \in \mathbb{R}$ and define

$$
\Omega:=\left\{x \in \mathbb{R}^{n}: \alpha<\rho_{F^{0}}(\bar{x}-x)<\beta\right\}
$$


for some $\beta>\alpha>0$. Then for each $\delta>0$ the function $S_{\delta}(x):=R_{\delta}\left(\rho_{F^{0}}(\bar{x}-x)\right)$ in (3.8) minimizes the integral (2.1) (here $\partial f(0)=[0, k]$ with $k>0)$ on $S_{\delta}(\cdot)+$ $W_{0}^{1,1}(\Omega)$ in virtue of the relations (3.10) and (3.14), where $w(\cdot)=S_{\delta}(\cdot), U=\Omega$, $\bar{u}(\cdot)$ is another minimizer, and the mapping $p(\cdot)$ is given by (3.13). Clearly, $\mu=\min _{x \in \bar{\Omega}} S_{\delta}(x)$, and it is enough only to choose $\delta>0, \alpha$ and $\beta$ such that $R_{\delta}(\alpha)>\mu$ and $R_{\delta}(r)=\mu$ for $\beta-\varepsilon \leq r \leq \beta$, where $\varepsilon>0$ is sufficiently small. Let, for instance, $\beta=2 \alpha$ and $k<\delta<2^{n-1} k$. Then for some $0<\varepsilon<\beta-\alpha$

$$
\delta\left(\frac{\alpha}{\alpha+\varepsilon}\right)^{n-1}>k, \quad \delta\left(\frac{\alpha}{2 \alpha-\varepsilon}\right)^{n-1}<k
$$

and, consequently,

$$
R_{\delta}(\alpha) \geq \mu+\int_{\alpha}^{\alpha+\varepsilon} \varphi\left(\delta\left(\frac{\alpha}{s}\right)^{n-1}\right) d s \geq \mu+\varphi\left(\delta\left(\frac{\alpha}{\alpha+\varepsilon}\right)^{n-1}\right) \varepsilon>\mu,
$$

while for $\beta-\varepsilon \leq r \leq \beta$ we have

$$
\mu \leq R_{\delta}(r) \leq \mu+\int_{\beta-\varepsilon}^{\beta} \varphi\left(\delta\left(\frac{\alpha}{s}\right)^{n-1}\right) d s \leq \mu+\varphi\left(\delta\left(\frac{\alpha}{2 \alpha-\varepsilon}\right)^{n-1}\right) \varepsilon=\mu .
$$

We use here the fact that $\varphi(t) \equiv 0$ on $[0, k]$ and $\varphi(t)>0$ for $t>k$. Thus $S_{\delta}(\cdot)$ is a nonconstant continuous minimizer of (2.1) admitting its minimum at each point $x \in \Omega$ with $\beta-\varepsilon \leq \rho_{F^{0}}(\bar{x}-x) \leq \beta$ that contradicts SMP.

As about the one-dimensional case, the function $L(\cdot)$ may have a nontrivial slope at zero, which, however, should be different from the slopes at all points $x \neq 0$. Let us give a simple example of such function, which, moreover, is neither strictly convex nor smooth near the origin.

Example 1 Fix an arbitrary strictly decreasing sequence $\left.\left\{\tau_{m}\right\} \subset\right] 0, \pi / 2[$ converging to zero, and define the continuous function $f:\left[0, \pi / 2\left[\rightarrow \mathbb{R}^{+}\right.\right.$, which is equal to tgt for $t=\tau_{m}, m=1,2, \ldots$, and for $\tau_{1}<t<\pi / 2$, and affine on each interval $] \tau_{m+1}, \tau_{m}[$. We set also $f(t)=+\infty$ for $t \geq \pi / 2$. Then $\partial f(0)=[-1,1]$ but, nevertheless, the Strong Maximum Principle is valid for the functional $\int_{\Omega} f\left(\left|u^{\prime}(x)\right|\right) d x$ due to Theorem 3 (see the condition (i)).

However, even in the case $n=1$ the function $f(\cdot)$ can not be affine near the origin. To see this it is enough to observe that if $f(t)=k t$ for $0 \leq t \leq \varepsilon$ then for each admissible function $u(\cdot) \in W^{1,1}(0,1)$ with the boundary values $u(0)=0$ and $u(1)=\varepsilon / 2$ we have by Jensen's inequality

$$
\int_{0}^{1} f\left(\left|u^{\prime}(x)\right|\right) d x \geq f\left(\left|\int_{0}^{1} u^{\prime}(x) d x\right|\right)=\frac{k \varepsilon}{2}=\int_{0}^{1} f\left(\left|\bar{u}^{\prime}(x)\right|\right) d x,
$$


where $\bar{u}(x):=\frac{\varepsilon}{2} x, x \in[0,1]$. On the other hand, the function $\tilde{u}(x)$ equal to 0 on $\left[0, \frac{1}{2}\right]$ and to $\varepsilon x-\frac{\varepsilon}{2}$ on $\left[\frac{1}{2}, 1\right]$ gives the same minimal value to the integral.

\section{The case $a>0$ : a one-point generalized Strong Maximum Principle}

As we have seen the traditional Strong Maximum Principle is no longer valid if $L=f \circ \rho_{F}$ with $\partial f^{*}(0) \neq\{0\}$. However, also in this case one can give a similar property (as explained in the end of Section 1), which clarifies the structure of minimizers of the functional (2.1) and has the same field of applications as the classical SMP. Let us start with the so-called one-point SMP when a test function admits a unique point of local minimum (maximum).

Setting $\hat{u}(x)=\mu+a \rho_{F^{0}}\left(x-x_{0}\right)$ with $\mu \in \mathbb{R}$ and $x_{0} \in \Omega$, we have already shown (see Corollary 2) that for an arbitrary minimizer $\bar{u}(\cdot)$ of (2.1) the inequality $\bar{u}(x) \geq \hat{u}(x)$ valid on a neighbourhood of $x_{0}$ becomes, in fact, an equality on another (possibly smaller) neighbourhood. First we show that this property can be extended to the maximal homothetic set $x_{0}+r F^{0}$ contained in $\Omega$.

Theorem 4 Assume that $L=f \circ \rho_{F}$, where $f: \mathbb{R}^{+} \rightarrow \mathbb{R}^{+} \cup\{+\infty\}$ is a convex lower semicontinuous function, $f(0)=0$ iff $t \in[0, a], a>0$, and $F \subset \mathbb{R}^{n}$ is a convex closed bounded set with $0 \in \operatorname{int} F$. Let $\Omega \subset \mathbb{R}^{n}$ be an open bounded region, $x_{0} \in \Omega$ and $\bar{u}(\cdot)$ be a continuous admissible minimizer of (2.1) on $u_{0}(\cdot)+W_{0}^{1,1}(\Omega)$. If the inequality

$$
\bar{u}(x) \geq \bar{u}\left(x_{0}\right)+a \rho_{F^{0}}\left(x-x_{0}\right)
$$

holds for each $x \in x_{0}+\mathfrak{r}^{+}\left(x_{0}\right) F^{0}$ then on this set the equality

$$
\bar{u}(x)=\bar{u}\left(x_{0}\right)+a \rho_{F^{0}}\left(x-x_{0}\right)
$$

takes place. Analogously, if

$$
\bar{u}(x) \leq \bar{u}\left(x_{0}\right)-a \rho_{F^{0}}\left(x_{0}-x\right)
$$

for each $x \in x_{0}-\mathfrak{r}^{-}\left(x_{0}\right) F^{0}$ then on the latter set the equality

$$
\bar{u}(x)=\bar{u}\left(x_{0}\right)-a \rho_{F^{0}}\left(x_{0}-x\right)
$$

holds.

Proof. We prove only the first assertion, while the second one is symmetric and can be proved similarly.

Let us define

$$
\begin{array}{r}
R:=\sup \left\{r>0: \bar{u}(x)=\bar{u}\left(x_{0}\right)+a \rho_{F^{0}}\left(x-x_{0}\right)\right. \\
\text { for all } \left.x \in x_{0}+r F^{0} \subset \Omega\right\} .
\end{array}
$$


By Corollary 2 we have $R>0$. Our goal now is to prove that $R=\mathfrak{r}^{+}\left(x_{0}\right)$. Assuming the contrary, i.e., $R<\mathfrak{r}^{+}\left(x_{0}\right)$ let us choose $\delta>0$ so small that

$$
R+\delta\left(\|F\|\left\|F^{0}\right\|+1\right)<\mathfrak{r}^{+}\left(x_{0}\right)
$$

and $\bar{x} \in \Omega$ with $R<\rho_{F^{0}}\left(\bar{x}-x_{0}\right)<R+\delta$ such that

$$
\bar{u}(\bar{x})>\bar{u}\left(x_{0}\right)+a \rho_{F^{0}}\left(\bar{x}-x_{0}\right)
$$

(see the condition (5.1)). By strictness of the inequalities (5.6) and (5.7) we find also $\varepsilon>0$ such that

$$
R+\delta\left(\|F\|\left\|F^{0}\right\|+1\right)+\varepsilon\|F\|\left\|F^{0}\right\|<\mathfrak{r}^{+}\left(x_{0}\right)
$$

and

$$
\bar{u}(\bar{x})>\bar{u}\left(x_{0}\right)+a\left(\rho_{F^{0}}\left(\bar{x}-x_{0}\right)+\varepsilon\right) .
$$

Setting $\mu:=\bar{u}\left(x_{0}\right)+a R$ let us define the function $\bar{v}(\cdot)$ to be equal to

$$
\max \{\bar{u}(x), \mu\}
$$

on $x_{0}+\mathfrak{r}^{+}\left(x_{0}\right) F^{0}$ and to $\bar{u}(x)$ on the rest of $\bar{\Omega}$. It is continuous because $R<\mathfrak{r}^{+}\left(x_{0}\right)$ and for each $x \in \Omega$ with $\rho_{F}\left(x-x_{0}\right) \geq R$ we have

$$
\bar{u}(x) \geq \bar{u}\left(x_{0}\right)+a \rho_{F^{0}}\left(x-x_{0}\right) \geq \mu,
$$

i.e., $\bar{v}(x)=\bar{u}(x)$. We claim that $\bar{v}(\cdot)$ is a minimizer of $(2.1)$ on $\bar{u}(\cdot)+W_{0}^{1,1}(\Omega)$. Indeed, it follows from $(5.10)$ that $\bar{v}(\cdot) \in \bar{u}(\cdot)+W_{0}^{1,1}(\Omega)$, and clearly $\nabla \bar{v}(x)=0$ for each $x \in \Omega$ with $\bar{v}(x) \neq \bar{u}(x)$. Denoting by $\Omega^{\prime}:=\{x \in \Omega: \bar{u}(x)=\bar{v}(x)\}$ we have

$$
\begin{aligned}
\int_{\Omega} f\left(\rho_{F}(\nabla \bar{v}(x))\right) d x & =\int_{\Omega^{\prime}} f\left(\rho_{F}(\nabla \bar{u}(x))\right) d x \leq \\
\int_{\Omega} f\left(\rho_{F}(\nabla \bar{u}(x))\right) d x & \leq \int_{\Omega} f\left(\rho_{F}(\nabla \bar{v}(x))\right) d x .
\end{aligned}
$$

Let $x_{0}^{\prime}$ be a unique point from $\left[x_{0}, \bar{x}\right]$ such that $\rho_{F^{0}}\left(x_{0}^{\prime}-x_{0}\right)=R$. Namely, $x_{0}^{\prime}=x_{0}+\lambda\left(\bar{x}-x_{0}\right)$ where $0<\lambda:=\frac{R}{\rho_{F^{0}}\left(\bar{x}-x_{0}\right)}<1$. Setting now $\beta:=$ $\rho_{F^{0}}\left(\bar{x}-x_{0}^{\prime}\right)+\varepsilon$, by $(5.9)$ we obtain

$$
\begin{aligned}
\bar{v}(\bar{x}) \geq & \bar{u}(\bar{x})>\bar{u}\left(x_{0}\right)+a \rho_{F^{0}}\left(x_{0}^{\prime}-x_{0}\right)+ \\
& a\left(\rho_{F^{0}}\left(\bar{x}-x_{0}^{\prime}\right)+\varepsilon\right)=\mu+a \beta .
\end{aligned}
$$

On the other hand, the inequality $\rho_{F^{0}}(\bar{x}-x) \leq \beta$ implies that

$$
\begin{aligned}
\rho_{F^{0}}\left(x-x_{0}\right) & \leq \rho_{F^{0}}(x-\bar{x})+\rho_{F^{0}}\left(\bar{x}-x_{0}^{\prime}\right)+\rho_{F^{0}}\left(x_{0}^{\prime}-x_{0}\right) \leq \\
& \leq\|F\|\left\|F^{0}\right\| \rho_{F^{0}}(\bar{x}-x)+\rho_{F^{0}}\left(\bar{x}-x_{0}^{\prime}\right)+R \leq \\
& \leq\left(\|F\|\left\|F^{0}\right\|+1\right) \rho_{F^{0}}\left(\bar{x}-x_{0}^{\prime}\right)+\varepsilon\|F\|\left\|F^{0}\right\|+R .
\end{aligned}
$$


Since obviously $\rho_{F^{0}}\left(\bar{x}-x_{0}^{\prime}\right)<\delta$, we can continue (5.13) and by (5.8) obtain that $\rho_{F^{0}}\left(x-x_{0}\right)<\mathfrak{r}^{+}\left(x_{0}\right)$. Hence, by the definition of $\bar{v}(\cdot)$,

$$
\bar{v}(x) \geq \mu \quad \forall x \in \bar{x}-\beta F^{0} .
$$

The inequalities (5.14) and (5.12) allow us to apply Theorem 1 with the minimizer $\bar{v}(\cdot)$ in the place of $\bar{u}(\cdot)$ and to find $\eta>0$ such that

$$
\bar{v}(x) \geq \mu+\varphi(\eta)\left(\beta-\rho_{F^{0}}(\bar{x}-x)\right) \quad \forall x \in \bar{x}-\beta F^{0} .
$$

In particular, for $x=x_{0}^{\prime} \in \bar{x}-\beta F^{0}$ we have

$$
\bar{v}\left(x_{0}^{\prime}\right) \geq \mu+\varphi(\eta) \varepsilon>\mu .
$$

However, by the definition of $R$ (see (5.5))

$$
\bar{u}\left(x_{0}^{\prime}\right)=\bar{u}\left(x_{0}\right)+a \rho_{F^{0}}\left(x_{0}^{\prime}-x_{0}\right)=\mu .
$$

So that $\bar{v}\left(x_{0}^{\prime}\right)=\mu$ as well, contradicting thus the strict inequality (5.15).

Whenever the gauge function $\rho_{F}(\cdot)$ is differentiable, the latter extremality result can be extended to arbitrary (densely) star-shaped domains in the place of $\rho_{F^{0}}$-balls $x_{0} \pm r F^{0}$.

Theorem 5 Assume that the lagrangean $L$ is such as in Theorem 4 with a convex compact and smooth set $F \subset \mathbb{R}^{n}, 0 \in \operatorname{int} F$; that $\Omega \subset \mathbb{R}^{n}$ is an open bounded region and $x_{0} \in \Omega$. Then for each continuous admissible minimizer $\bar{u}(\cdot)$ of (2.1) on $u_{0}(\cdot)+W_{0}^{1,1}(\Omega)$ and each open $\hat{\Omega} \subset \Omega$, which is densely starshaped w.r.t. $x_{0}$, the inequality (5.1) (respectively, (5.3)) holds for all $x \in \hat{\Omega}$ only if the respective equality (5.2) (or (5.4)) is valid on $\hat{\Omega}$.

Proof. As earlier we prove here only the implication (5.1) $\Longrightarrow(5.2)$, while the other one $((5.3) \Longrightarrow(5.4))$ can be treated similarly. Moreover, it is enough to prove the equality (5.2) on the star $\operatorname{St}_{\hat{\Omega}}\left(x_{0}\right)$, because to each $x \in \hat{\Omega} \backslash \operatorname{St}_{\hat{\Omega}}\left(x_{0}\right) \subset$ $\overline{\mathrm{St}_{\hat{\Omega}}\left(x_{0}\right)}$ it can be extended by continuity of the involved functions.

So, let us assume validity of (5.1) on $\hat{\Omega}$ and fix $\bar{x} \in \operatorname{St}_{\hat{\Omega}}\left(x_{0}\right)$. By the compactness argument we choose $\varepsilon>0$ such that

$$
\left[x_{0}, \bar{x}\right] \pm \varepsilon F^{0} \subset \hat{\Omega} .
$$

Set

$$
\delta:=2 \varepsilon \mathfrak{M}_{F^{0}}\left(\frac{2 \varepsilon}{\Delta} ; \frac{\varepsilon}{\varepsilon+\Delta}, \frac{\Delta}{\varepsilon+\Delta}\right),
$$

where $\mathfrak{M}_{F^{0}}$ is the rotundity modulus defined by (2.5) and $\Delta$ is the $\rho_{F^{0}}$-diameter of the domain $\hat{\Omega}$, i.e.,

$$
\Delta:=\sup _{\xi, \eta \in \hat{\Omega}} \rho_{F^{0}}(\xi-\eta)>0 .
$$

It follows from the remarks of Section 2 that $F^{0}$ is rotund, and therefore $\delta>0$. 
Let us consider an uniform partition of the segment $\left[x_{0}, \bar{x}\right]$ by the points $x_{i}:=x_{0}+i h \frac{\bar{x}-x_{0}}{\rho_{F 0}\left(\bar{x}-x_{0}\right)}, i=0,1, \ldots, m$, with

$$
h:=\frac{\rho_{F^{0}}\left(\bar{x}-x_{0}\right)}{m} \leq \min \left\{\frac{\varepsilon}{\sqrt{M}}, \frac{\delta}{M}\right\},
$$

where $M:=\left(\|F\|\left\|F^{0}\right\|+1\right)^{2}$. Since the inequality (5.1) holds for all $x \in$ $x_{0}+\varepsilon F^{0}$ (see (5.16)) and $\rho_{F^{0}}\left(x_{1}-x_{0}\right)=h \leq \frac{\varepsilon}{\|F\|\left\|F^{0}\right\|+1}$, it follows from Corollary 2 that

$$
\bar{u}\left(x_{1}\right)=\bar{u}\left(x_{0}\right)+a \rho_{F^{0}}\left(x_{1}-x_{0}\right)=\bar{u}\left(x_{0}\right)+a h .
$$

We want to prove by induction in $i$ that

$$
\bar{u}\left(x_{i}\right)=\bar{u}\left(x_{0}\right)+i a h,
$$

$i=1,2, \ldots, m$. Then for $i=m$ we have

$$
\bar{u}(\bar{x})=\bar{u}\left(x_{m}\right)=\bar{u}\left(x_{0}\right)+a \rho_{F^{0}}\left(\bar{x}-x_{0}\right),
$$

and Theorem will be proved due to arbitrarity of $\bar{x} \in \mathrm{St}_{\hat{\Omega}}\left(x_{0}\right)$.

So, we assume that (5.20) is true for some $i$ with $1 \leq i \leq m-1$ and define the function $\bar{u}_{i}: \bar{\Omega} \rightarrow \mathbb{R}$ as

$$
\max \left\{\bar{u}(x), \min \left\{\bar{u}\left(x_{i}\right)+a \rho_{F^{0}}\left(x-x_{i}\right), \mu_{i}-a \rho_{F^{0}}\left(x_{i}-x\right)\right\}\right\}
$$

on $\hat{\Omega}$ and as $\bar{u}(x)$ elsewhere. Here

$$
\mu_{i}:=\bar{u}\left(x_{0}\right)+a(i+M) h .
$$

Let us devide the remainder of the proof in three steps.

Step 1. We claim first that for each $x \notin\left[x_{0}, \bar{x}\right] \pm \varepsilon F^{0}$ the inequality

$$
\rho_{F^{0}}\left(x-x_{0}\right)+\rho_{F^{0}}\left(x_{i}-x\right)-\rho_{F^{0}}\left(x_{i}-x_{0}\right) \geq \delta
$$

holds. Indeed, given such a point $x$ we have

$$
\rho_{0}:=\rho_{F^{0}}\left(x-x_{0}\right) \geq \varepsilon \text { and } \rho_{i}:=\rho_{F^{0}}\left(x_{i}-x\right) \geq \varepsilon .
$$

On the other hand, clearly $\rho_{0} \leq \Delta$ and $\rho_{i} \leq \Delta$ (see (5.18)). Hence, by the monotonicity

$$
\lambda:=\frac{\rho_{0}}{\rho_{0}+\rho_{i}} \in\left[\frac{\varepsilon}{\Delta+\varepsilon}, \frac{\Delta}{\Delta+\varepsilon}\right] .
$$

Furthermore,

$$
\begin{aligned}
& \rho_{F^{0}}\left(x-x_{0}\right)+\rho_{F^{0}}\left(x_{i}-x\right)-\rho_{F^{0}}\left(x_{i}-x_{0}\right)= \\
= & \left(\rho_{0}+\rho_{i}\right)\left[1-\rho_{F^{0}}\left(\frac{\rho_{i}}{\rho_{0}+\rho_{i}} \frac{x_{i}-x}{\rho_{i}}+\frac{\rho_{0}}{\rho_{0}+\rho_{i}} \frac{x-x_{0}}{\rho_{0}}\right)\right] \geq \\
\geq & 2 \varepsilon\left[1-\rho_{F^{0}}(\xi+\lambda(\eta-\xi))\right],
\end{aligned}
$$


where $\xi:=\frac{x_{i}-x}{\rho_{i}}$ and $\eta:=\frac{x-x_{0}}{\rho_{0}}$. Since

$$
\xi-\eta=\left(\frac{1}{\rho_{0}}+\frac{1}{\rho_{i}}\right)\left(\frac{\rho_{0}}{\rho_{0}+\rho_{i}} x_{i}+\frac{\rho_{i}}{\rho_{0}+\rho_{i}} x_{0}-x\right),
$$

by the choice of $x$ we obtain

$$
\rho_{F^{0}}(\xi-\eta) \geq\left(\frac{1}{\rho_{0}}+\frac{1}{\rho_{i}}\right) \varepsilon \geq \frac{2 \varepsilon}{\Delta}
$$

Joining together (5.24)-(5.26), (5.17) and the definition of the rotundity modulus (2.5) we immediately arrive at (5.23).

Step 2. Let us show that $\bar{u}_{i}(\cdot)$ is the continuous minimizer of the functional (2.1) on $\bar{u}(\cdot)+W_{0}^{1,1}(\Omega)$. Given $x \in \hat{\Omega}$ with $x \notin\left[x_{0}, \bar{x}\right] \pm \varepsilon F^{0}$ it folows from (5.23) and (5.19) that

$$
\rho_{F^{0}}\left(x-x_{0}\right)+\rho_{F^{0}}\left(x_{i}-x\right) \geq \delta+i h \geq(i+M) h .
$$

Since $\bar{u}(x) \geq \bar{u}\left(x_{0}\right)+a \rho_{F^{0}}\left(x-x_{0}\right)$ by the condition, we deduce from (5.27) and (5.22) that $\bar{u}(x) \geq \mu_{i}-a \rho_{F^{0}}\left(x_{i}-x\right)$. Consequently, $\bar{u}_{i}(x)=\bar{u}(x)(\operatorname{see}(5.21))$. Due to (5.16) this means continuity of the function $\bar{u}_{i}(\cdot)$. Besides that obviously $\bar{u}_{i}(\cdot) \in \bar{u}(\cdot)+W_{0}^{1,1}(\Omega)$. We see from (5.21) that for each $x \in \Omega$ with $\bar{u}_{i}(x) \neq$ $\bar{u}(x)$ the gradient $\nabla \bar{u}_{i}(x)$ belongs to $a F$, and therefore $f\left(\rho_{F}\left(\nabla \bar{u}_{i}(x)\right)\right)=0$. The same argument as in the proof of Theorem 4 (see (5.11)) convinces us that $\bar{u}_{i}(\cdot)$ gives the minimum to $(2.1)$ among all the functions with the same boundary data.

Step 3. Here we prove that for each $x$ with

$$
\rho_{F^{0}}\left(x-x_{i}\right) \leq\left(\|F\|\left\|F^{0}\right\|+1\right) h
$$

(such $x$ belong to $\hat{\Omega}$ by (5.16) and (5.19)) the inequality

$$
\bar{u}_{i}(x) \geq \bar{u}_{i}\left(x_{i}\right)+a \rho_{F^{0}}\left(x-x_{i}\right)
$$

holds. Indeed, it follows from (5.28) and (2.3) that

$$
\rho_{F^{0}}\left(x-x_{i}\right)+\rho_{F^{0}}\left(x_{i}-x\right) \leq M h,
$$

and taking into account the definition of $\mu_{i}$ (see (5.22)) and the induction hypothesis (5.20) we obtain from (5.30):

$$
\bar{u}\left(x_{i}\right)+a \rho_{F^{0}}\left(x-x_{i}\right) \leq \mu_{i}-a \rho_{F^{0}}\left(x_{i}-x\right),
$$

i.e., the minimum in $(5.21)$ is equal to $\bar{u}\left(x_{i}\right)+a \rho_{F^{0}}\left(x-x_{i}\right)$. This implies (5.29) because

$$
\bar{u}_{i}\left(x_{i}\right)=\max \left\{\bar{u}\left(x_{i}\right), \min \left\{\bar{u}\left(x_{i}\right), \mu_{i}\right\}\right\}=\bar{u}\left(x_{i}\right)
$$


Applying now Corollary 2 to the minimizer $\bar{u}_{i}(\cdot)$ (see Step 2) and to the point $x_{i} \in \Omega$ we conclude that the inequality (5.29) becomes the equality for each $x \in x_{i}+h F^{0}$, in particular, for $x=x_{i+1}$. Thus, by (5.31) and (5.20) we have

$$
\bar{u}_{i}\left(x_{i+1}\right)=\bar{u}\left(x_{0}\right)+(i+1) a h .
$$

On the other hand, by the definition of $\bar{u}_{i}(\cdot)$ (see $(5.21)$ )

$$
\bar{u}\left(x_{i+1}\right) \leq \bar{u}_{i}\left(x_{i+1}\right)=\bar{u}\left(x_{0}\right)+a \rho_{F^{0}}\left(x_{i+1}-x_{0}\right) .
$$

Since the opposite inequality holds by the condition, we finally obtain

$$
\begin{aligned}
\bar{u}\left(x_{i+1}\right) & =\bar{u}\left(x_{0}\right)+a \rho_{F^{0}}\left(x_{i+1}-x_{0}\right)= \\
& =\bar{u}\left(x_{0}\right)+(i+1) a h,
\end{aligned}
$$

and the induction is complete.

The following one-point version of SMP is the immediate consequence of the latter result.

Corollary 3 (one-point generalized Strong Maximum Principle) Let the lagrangean $L=f \circ \rho_{F}$ be such as in Theorem 5 (the set $F$ is supposed to be smooth), and $\Omega \subset \mathbb{R}^{n}$ be an open bounded and densely star-shaped w.r.t. $x_{0} \in \Omega$. Then given a continuous admissible minimizer $\bar{u}(\cdot)$ of (2.1) on $u_{0}(\cdot)+W_{0}^{1,1}(\Omega)$ the inequality (5.1) (respectively, (5.3)) holds for all $x \in \Omega$ if and only if the respective equality (5.2) (or (5.4)) takes place.

Notice that the (dense) star-shapeness of the region $\Omega$ in the above statement can not be dropped as the following simple counter-example shows.

Example 2 Let $\Sigma \subset \mathbb{R}^{n}$ be an arbitrary open bounded set, which is starshaped w.r.t. $x_{0} \in \Sigma$. Fix $\bar{x} \in \Sigma, \bar{x} \neq x_{0}$, and a real number $0<r<$ $\min \left\{\left\|x_{0}-\bar{x}\right\|, \mathrm{d}_{\partial \Sigma}(\bar{x})\right\}$, where $\mathrm{d}_{\partial \Sigma}(\cdot)$ means the distance from a point to the boundary of $\Sigma$. Let us consider the other domain $\Omega:=\Sigma \backslash(\bar{x}+r \bar{B})$, where $\bar{B}$ is the closed unit ball centred in zero. Assuming the function $f: \mathbb{R}^{+} \rightarrow \mathbb{R}^{+} \cup\{+\infty\}$ and the gauge set $F \subset \mathbb{R}^{n}$ to be as in Theorem 5 we construct a continuous admissible minimizer $\bar{u}(\cdot)$ of (2.1) with the property

$$
\bar{u}(x) \geq \bar{u}\left(x_{0}\right)+a \rho_{F^{0}}\left(x-x_{0}\right) \quad \forall x \in \Omega,
$$

where the inequality is strict on some nonempty (open) set $\Omega^{\prime} \subset \Omega$.

In what follows by $\mathrm{St}\left(x_{0}\right)$ we intend the star associated with the point $x_{0}$ in the domain $\Omega$. It is obvious that $x \in \Omega \backslash$ St $\left(x_{0}\right)$ if and only if the segment $\left[x_{0}, x\right]$ meets the ball $\bar{x}+r \bar{B}$, or, in other words, the quadratic equation

$$
\begin{aligned}
& \left\|x_{0}+\lambda\left(x-x_{0}\right)-\bar{x}\right\|^{2}= \\
& \lambda^{2}\left\|x-x_{0}\right\|^{2}-2 \lambda\left\langle x-x_{0}, \bar{x}-x_{0}\right\rangle+\left\|\bar{x}-x_{0}\right\|^{2}=r^{2}
\end{aligned}
$$


has (one or two) roots both belonging to the interval $] 0,1[$. We write the condition of resolvability of (5.33) as

$$
\mathfrak{D}(x):=\left\langle x-x_{0}, \bar{x}-x_{0}\right\rangle^{2}-\left\|x-x_{0}\right\|^{2}\left(\left\|\bar{x}-x_{0}\right\|^{2}-r^{2}\right) \geq 0,
$$

and denote by

$$
\lambda_{ \pm}(x):=\frac{\left\langle x-x_{0}, \bar{x}-x_{0}\right\rangle \pm \sqrt{\mathfrak{D}(x)}}{\left\|x-x_{0}\right\|^{2}}
$$

its roots. Taking into account the continuity of all the involved functions we can represent:

$$
\begin{array}{r}
\Omega^{\prime}:=\operatorname{int}\left(\Omega \backslash \operatorname{St}\left(x_{0}\right)\right)=\{x \in \Sigma: \mathfrak{D}(x)>0 \text { and } \\
\left.0<\lambda_{-}(x)<\lambda_{+}(x)<1\right\}
\end{array}
$$

and

$$
\begin{aligned}
& E:=\partial\left(\Omega \backslash \operatorname{St}\left(x_{0}\right)\right) \cap \partial\left(\operatorname{St}\left(x_{0}\right)\right)= \\
& \left\{x \in \Sigma: \mathfrak{D}(x)=0 \text { and } 0<\lambda_{ \pm}(x)=\frac{\left\langle x-x_{0}, \bar{x}-x_{0}\right\rangle}{\left\|x-x_{0}\right\|^{2}}<1\right\} .
\end{aligned}
$$

The open set $\Omega^{\prime}$ is clearly nonempty because it contains, e.g., all the points $\bar{x}+(r+\delta) \frac{\bar{x}-x_{0}}{\left\|\bar{x}-x_{0}\right\|}$ with $\delta>0$ small enough.

For each $x \in E$ let us define the "trace" operator

$$
\Phi(x):=x_{0}+\lambda_{ \pm}(x)\left(x-x_{0}\right),
$$

which is continuous and satisfies the following "cone" property: if $x \in E$ and $x^{\prime}=x_{0}+\lambda\left(x-x_{0}\right) \in \Omega$ with $\lambda>\lambda_{ \pm}(x)$ then also $x^{\prime} \in E$ and $\Phi\left(x^{\prime}\right)=$ $\Phi(x)$. Indeed, we find from (5.34) and (5.35) that $\mathfrak{D}\left(x^{\prime}\right)=\lambda^{2} \mathfrak{D}(x)=0$ and $\left.\lambda_{ \pm}\left(x^{\prime}\right)=\frac{1}{\lambda} \lambda_{ \pm}(x) \in\right] 0,1\left[\right.$. Hence $x^{\prime} \in E$ and the equality $\Phi\left(x^{\prime}\right)=\Phi(x)$ follows directly from (5.38). This property and continuity of $\Phi(\cdot)$ imply that the image $C:=\Phi(E)$ is a compact subset of $\{x \in \Omega:\|x-\bar{x}\|=r\}$. Define the function $\bar{u}: \bar{\Omega} \rightarrow \mathbb{R}$ by the formula

$$
\bar{u}(x):=\left\{\begin{array}{ccc}
a \inf _{y \in C}\left\{\rho_{F^{0}}(x-y)+\rho_{F^{0}}\left(y-x_{0}\right)\right\} & \text { if } & x \in \bar{\Omega} \backslash \operatorname{St}\left(x_{0}\right) ; \\
a \rho_{F^{0}}\left(x-x_{0}\right) & \text { if } & x \in \operatorname{St}\left(x_{0}\right),
\end{array}\right.
$$

and show, first, its continuity. To this end it is enough to verify the equality

$$
\inf _{y \in C}\left\{\rho_{F^{0}}(x-y)+\rho_{F^{0}}\left(y-x_{0}\right)\right\}=\rho_{F^{0}}\left(x-x_{0}\right)
$$

for each point $x \in E$. Notice that the inequality " $\geq "$ in (5.40) is obvious. In order to prove " $\leq$ " let us take an arbitrary $x \in E$ and put $y:=\Phi(x)=$ $x_{0}+\lambda_{ \pm}(x)\left(x-x_{0}\right)$. Then $\rho_{F^{0}}\left(y-x_{0}\right)=\lambda_{ \pm}(x) \rho_{F^{0}}\left(x-x_{0}\right)$ and $\rho_{F^{0}}(x-y)=$ 
$\left(1-\lambda_{ \pm}(x)\right) \rho_{F^{0}}\left(x-x_{0}\right)$. So that the inequality " $\leq "$ in (5.40) immediately follows. The function $\bar{u}(\cdot)$ is, moreover, lipschitzean on $\bar{\Omega}$, and its Clarke's subdifferential $\partial^{c} \bar{u}(x)$ is always contained in $a F$ (see [4, p.92]). Since by Rademaher's Theorem the gradient $\nabla \bar{u}(x)$ almost everywhere exists and $\nabla \bar{u}(x) \in \partial^{c} \bar{u}(x)$, we have $f\left(\rho_{F}(\nabla \bar{u}(x))\right)=0$ for a.e. $x \in \Omega$, and, consequently, $\bar{u}(\cdot)$ is a minimizer in the problem (1.1).

The inequality (5.32) is obvious (here $\bar{u}\left(x_{0}\right)=0$ ). Fix now $x \in \Omega^{\prime}$ and $y \in C=\Phi(E)$. Then $y=x_{0}+\lambda_{ \pm}(z)\left(z-x_{0}\right)$ with $z \in E$. Assuming that there exists $\lambda>0$ with $x-y=\lambda\left(y-x_{0}\right)$ and taking into account the representation of $y$, we have $x=x_{0}+\mu\left(z-x_{0}\right)$ with $\mu:=(\lambda+1) \lambda_{ \pm}(z)>\lambda_{ \pm}(z)$. Due to the "cone" property of the "trace" operator (see above) $x \in E$ as well, which is a contradiction. Thus, by the rotundity of the set $F^{0}$ the strict inequality

$$
\rho_{F^{0}}(x-y)+\rho_{F^{0}}\left(y-x_{0}\right)>\rho_{F^{0}}\left(x-x_{0}\right)
$$

holds. Finally, by the compactness of the set $C$ and by arbitrarity of $y \in$ $C$ we conclude that the inequality (5.32) is strict whenever $x \in \Omega^{\prime}$, and the construction is complete.

The method used in Example 2 can be essentially sharpened in order to cover the case of an arbitrary domain $\Omega$, in which an open subset is not linearily attainable from $x_{0} \in \Omega$. So, let us formulate the following conjecture.

Conjecture The condition $\Omega \subset \overline{\mathrm{St}\left(x_{0}\right)}$ is necessary for validity of the one-point Strong Maximum Principle w.r.t. $x_{0} \in \Omega$ as given by Corollary 3.

\section{A multi-point version of the Strong Maximum Principle}

It follows from Theorem 5 that the one-point version of SMP given in the previous section (see Corollary 3) can be easily extended to the case when the test function $\hat{u}(\cdot)$ has a finite number of local minimum (maximum) points. Namely, let $x_{i} \in \mathbb{R}^{n}, i=1, \ldots, m$, be different, $m \in \mathbb{N}$, and arbitrary real numbers $\theta_{1}, \theta_{2}, \ldots, \theta_{m}$ be such that the compatibility condition

$$
\theta_{i}-\theta_{j}<a \rho_{F^{0}}\left(x_{i}-x_{j}\right), i \neq j,
$$

is fulfiled. Considering the functions

$$
\hat{u}^{+}(x):=\min _{1 \leq i \leq m}\left\{\theta_{i}+a \rho_{F^{0}}\left(x-x_{i}\right)\right\}
$$

and

$$
\hat{u}^{-}(x):=\max _{1 \leq i \leq m}\left\{\theta_{i}-a \rho_{F^{0}}\left(x_{i}-x\right)\right\},
$$


we deduce immediately from (6.1) that $\hat{u}^{+}\left(x_{i}\right)=\hat{u}^{-}\left(x_{i}\right)=\theta_{i}, i=1, \ldots, m$, and that $\left\{x_{1}, x_{2}, \ldots, x_{m}\right\}$ is the set of all local minimum (maximum) points of the function (6.2) or (6.3), respectively.

Before proving of the main statement of this section let us formulate some property of the minimizers in (6.2) (respectively, of the maximizers in (6.3)). It is given in a more general setting and generalizes a well-known property of metric projections.

Lemma 1 Let $\Gamma \subset \mathbb{R}^{n}$ be a nonempty closed set and $\theta(\cdot)$ be a real-valued function defined on $\Gamma$. Given $x \in \mathbb{R}^{n} \backslash \Gamma$ assume that the minimum of the function $y \mapsto \theta(y)+a \rho_{F^{0}}(x-y)$ (respectively, the maximum of $y \mapsto \theta(y)-$ $a \rho_{F^{0}}(y-x)$ ) on $\Gamma$ is attained at some point $\bar{y} \in \Gamma$. Then $\bar{y}$ continues to be a minimizer of $y \mapsto \theta(y)+a \rho_{F^{0}}\left(x_{\lambda}-y\right)$ (respectively, a maximizer of $y \mapsto$ $\left.\theta(y)-a \rho_{F^{0}}\left(y-x_{\lambda}\right)\right)$ for all $\lambda \in[0,1]$, where $x_{\lambda}:=(1-\lambda) x+\lambda \bar{y}$.

Proof. Given $\lambda \in[0,1]$ and $y \in \Gamma$ we obviously have $x-y=x_{\lambda}-y+\lambda(x-\bar{y})$, and by the semilinearity

$$
\rho_{F^{0}}(x-y) \leq \rho_{F^{0}}\left(x_{\lambda}-y\right)+\lambda \rho_{F^{0}}(x-\bar{y}) .
$$

Since $\theta(\bar{y})+a \rho_{F^{0}}(x-\bar{y}) \leq \theta(y)+a \rho_{F^{0}}(x-y)$, it follows from (6.4) that

$$
\theta(\bar{y})+a(1-\lambda) \rho_{F^{0}}(x-\bar{y}) \leq \theta(y)+a \rho_{F^{0}}\left(x_{\lambda}-y\right) .
$$

This proves the first assertion because $(1-\lambda)(x-\bar{y})=x_{\lambda}-\bar{y}$. The second case can be easily reduced to the first one by changing the signs.

Theorem 6 (multi-point version of SMP) Assume that the lagrangean $L$ $=f \circ \rho_{F}$ satisfies our standing hypotheses with the smooth gauge set $F$, and $\Omega \subset \mathbb{R}^{n}$ is an open bounded convex region containing the points $x_{1}, \ldots, x_{m}$. Then each continuous admissible minimizer $\bar{u}(\cdot)$ in the variational problem (1.1) such that $\bar{u}\left(x_{i}\right)=\theta_{i}, i=1, \ldots, m$, and

$$
\bar{u}(x) \geq \hat{u}^{+}(x) \quad \forall x \in \Omega
$$

(respectively, $\bar{u}(x) \leq \hat{u}^{-}(x) \forall x \in \Omega$ ) coincides with $\hat{u}^{+}(x)$ (respectively, with $\left.\hat{u}^{-}(x)\right)$. Here the functions $\hat{u}^{ \pm}(\cdot)$ are defined by (6.2) and (6.3).

Proof. As usual we prove only the first part of the statement. Denoting by

$$
C_{i}:=\left\{x \in \Omega: \hat{u}^{+}(x)=\theta_{i}+a \rho_{F^{0}}\left(x-x_{i}\right)\right\},
$$

and by $\Omega_{i}:=\operatorname{int} C_{i}, i=1, \ldots, m$, we obviously have $x_{i} \in \Omega_{i}$ and deduce from Lemma 1 that the open set $\Omega_{i}$ is star-shaped w.r.t. $x_{i}$. Since on $\Omega_{i}$ the inequality (6.5) admits the form

$$
\bar{u}(x) \geq \bar{u}\left(x_{i}\right)+a \rho_{F^{0}}\left(x-x_{i}\right),
$$


applying Theorem 5 we conclude that

$$
\bar{u}(x)=\bar{u}\left(x_{i}\right)+a \rho_{F^{0}}\left(x-x_{i}\right)=\hat{u}^{+}(x) \quad \forall x \in \Omega_{i} .
$$

It is enough now to observe that the union of (disjoint) sets $\Omega_{i}, i=1, \ldots, m$, is dense in $\Omega$. Therefore, the equality $\bar{u}(x)=\hat{u}^{+}(x)$ holds for all $x \in \Omega$ due to the continuity of both functions.

\section{References}

[1] A. Cellina, On the Strong Maximum Principle, Proc. Amer. Math. Soc. 130 (2002), 413-418.

[2] A. Cellina, On minima of a functional of the gradient: sufficient conditions, Nonlin. Anal.: Theory, Meth. and Appl. 20 (1993), 343-347.

[3] A. Cellina, C. Mariconda and G. Treu, Comparison results without strict convexity, Disc. and Cont. Dynam. Syst.- Series B 11 (2009), 57-65.

[4] F.H. Clarke, Optimization and Nonsmooth Analysis, Wiley, New York, 1983.

[5] G. Colombo and P. Wolenski, Variational Analysis for a class of minimal time functions in Hilbert spaces, J. Convex Anal. 11 (2004), 335-361.

[6] D. Gilbarg and N. Trudinger, Elliptic Partial Differential Equations of Second Order, Springer, New York, 1998.

[7] D. Kindelehrer and G. Stampachia, An Introduction to Variational Inequalities and their Applications, Academic Press, New York, 1980.

[8] R. R. Phelps, Convex Functions, Monotone Operators and Differentiability, Lecture Notes in Math. 1364, Springer, New York, 1989.

[9] P. Pucci, J. Serrin, The strong maximum principle revisited, J. Dif. Equat. 196 (2004), 1-66.

[10] P. Pucci, J. Serrin, The Strong Maximum Principle, Progress in Nonlinear Differential Equations and their Applications.Vol. 73, Birkhauser, Switzerland, 2007.

[11] R. T. Rockafellar, Convex Analysis, Princeton University Press, Princeton, New York, 1972. 